\title{
Microbial Polyphenol Production in a Biphasic Process
}

\author{
Apilaasha Tharmasothirajan $^{\dagger, \star}$, Marie Wellfonder ${ }^{\dagger}$, Jan Marienhagen ${ }^{\dagger, t^{*}}$ \\ †Institute of Bio- and Geosciences, IBG-1: Biotechnology, Forschungszentrum Jülich, D-52425 Jülich, \\ Germany \\ \#nstitute of Biotechnology, RWTH Aachen University, Worringer Weg 3, D-52074 Aachen, Germany \\ ${ }^{*}$ Corresponding author: \\ Prof. Dr. Jan Marienhagen, phone: +49 246161 2843, \\ e-mail: j.marienhagen@,fz-juelich.de; ORCID ID: 0000-0001-5513-3730
}

This file includes:

Number of pages: 7

Number of tables: 2

Numer of figures: 5 


\section{Supporting Information}

A
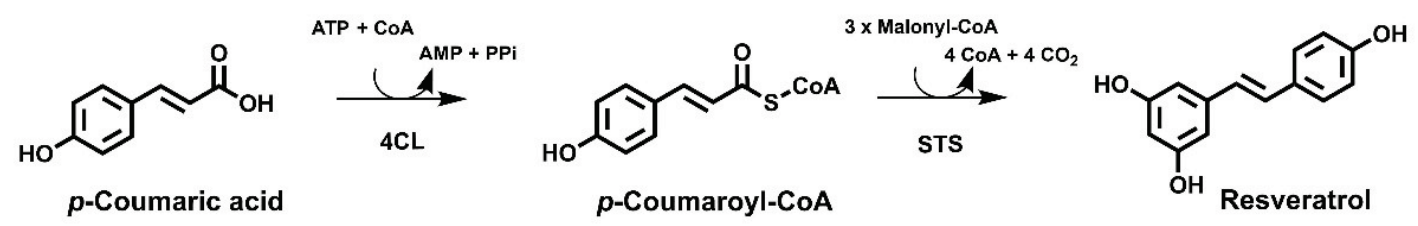

B

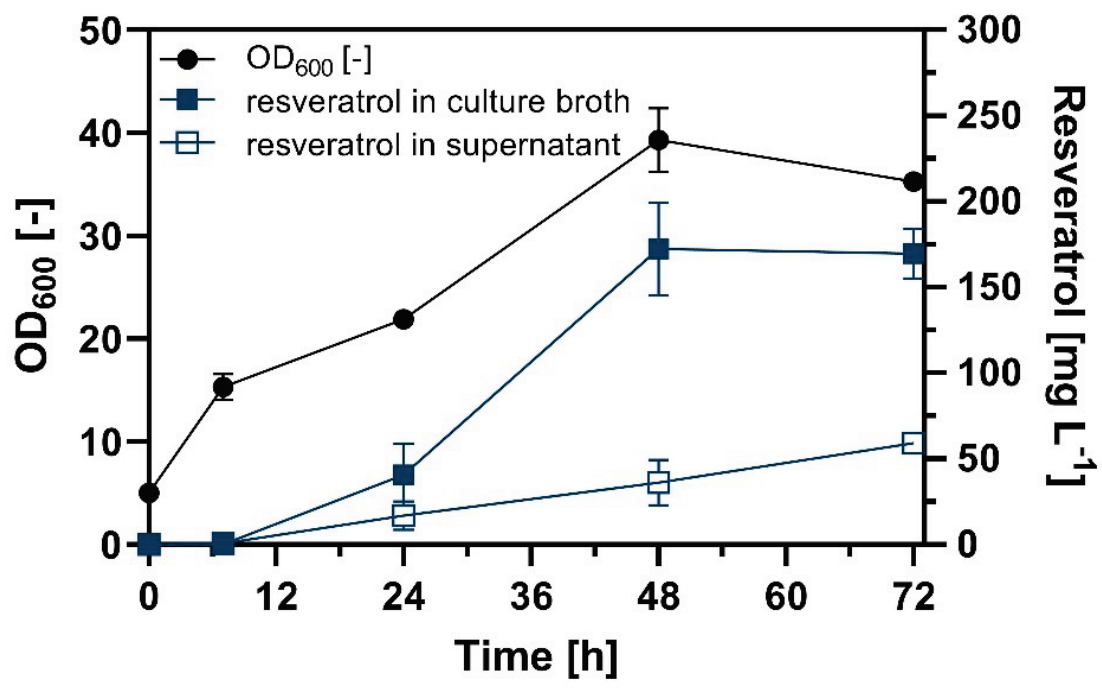

Figure S1. A: RES synthesis from $p$-CA. $p$-CA is CoA-activated by a 4-coumaroyl-CoA ligase yielding p-coumaroyl-CoA. Subsequently, a stilbene synthase catalyzes the iterative condensation of three acetyl units (derived from malonyl-CoA) to the CoA-linked starter molecule. B: RES production in C. glutamicum-RES. Cultivation conditions: defined CGXII medium containing $220 \mathrm{mM}$ D-glucose and $5 \mathrm{Mm} p$-CA, initial $\mathrm{pH}_{0}=7$, $\mathrm{T}=30{ }^{\circ} \mathrm{C}, \mathrm{n}=1000 \mathrm{rpm}$, shaking diameter $=3 \mathrm{~mm} ; \mathrm{V}=2000 \mu \mathrm{L}, \mathrm{V}_{\mathrm{L}}=900 \mu \mathrm{L}$ per well. Data represent average values and standard deviation of three biological replicates. 

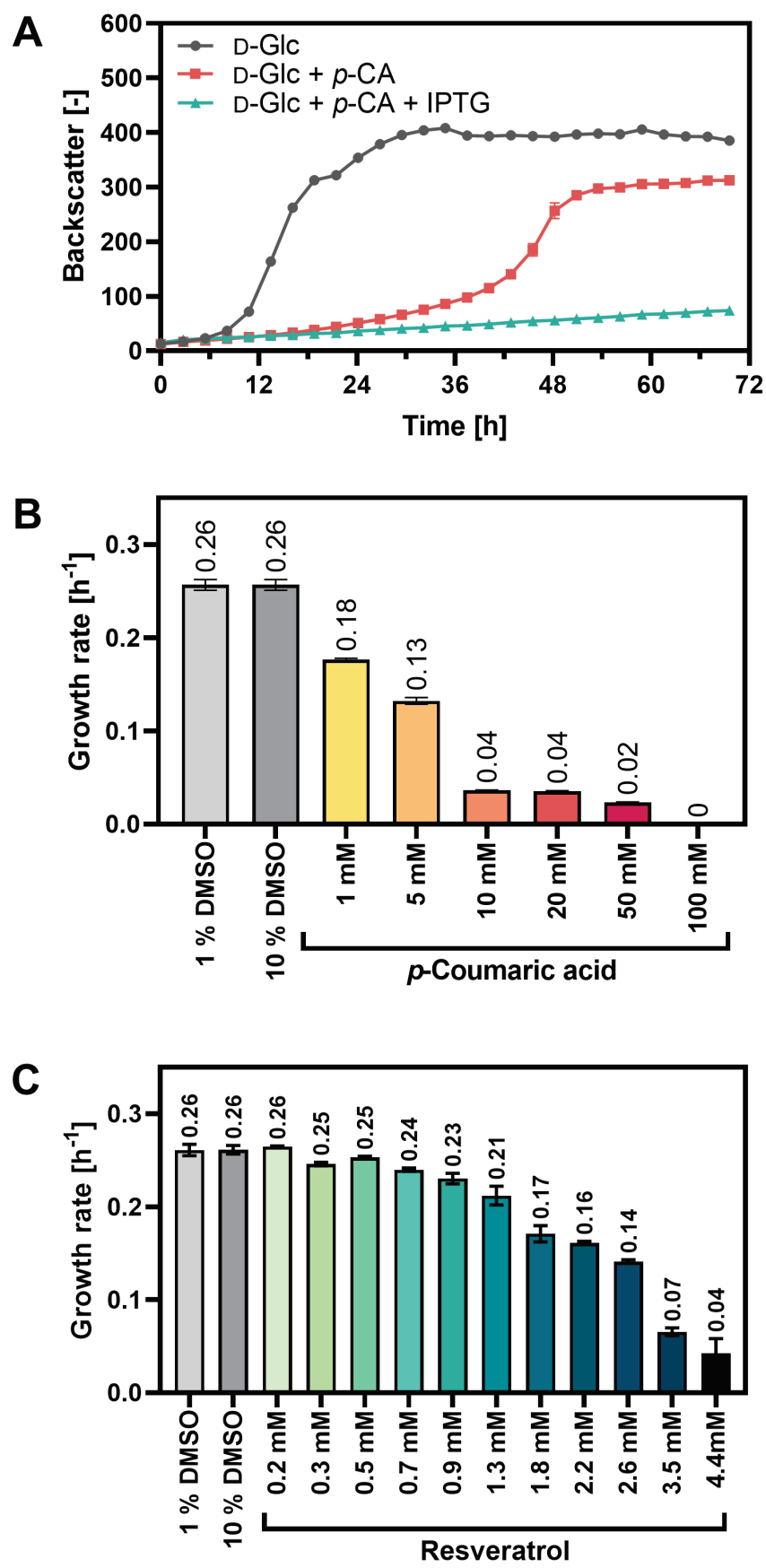

Figure S2: Effect of RES and $p$-CA on growth of $C$. glutamicum-RES. A: Impact of supplemented $p$-CA and RES production of $C$. glutamicum-RES on growth during cultivations in defined CGXII medium. B+C: Growth rates of C. glutamicum-RES on different concentrations of (B) p-CA $(0-100 \mathrm{mM})$ and (C) RES (0- $4.4 \mathrm{mM})$. Cultivations were conducted in microtiter flowerplates. Biomass formation was followed by measuring the backscattered light intensity (gain 10) at a wavelength of $620 \mathrm{~nm}$. Cultivation conditions: initial $\mathrm{pH}_{0}=7, \mathrm{~T}=30^{\circ} \mathrm{C}$, $\mathrm{n}=1,000 \mathrm{rpm}$, shaking diameter $=3 \mathrm{~mm} ; \mathrm{V}=2,000 \mu \mathrm{L}, \mathrm{V}_{\mathrm{L}}=900 \mu \mathrm{L}$ per well. Data represent average values and standard deviation of three biological replicates. 


\section{Supporting Information}
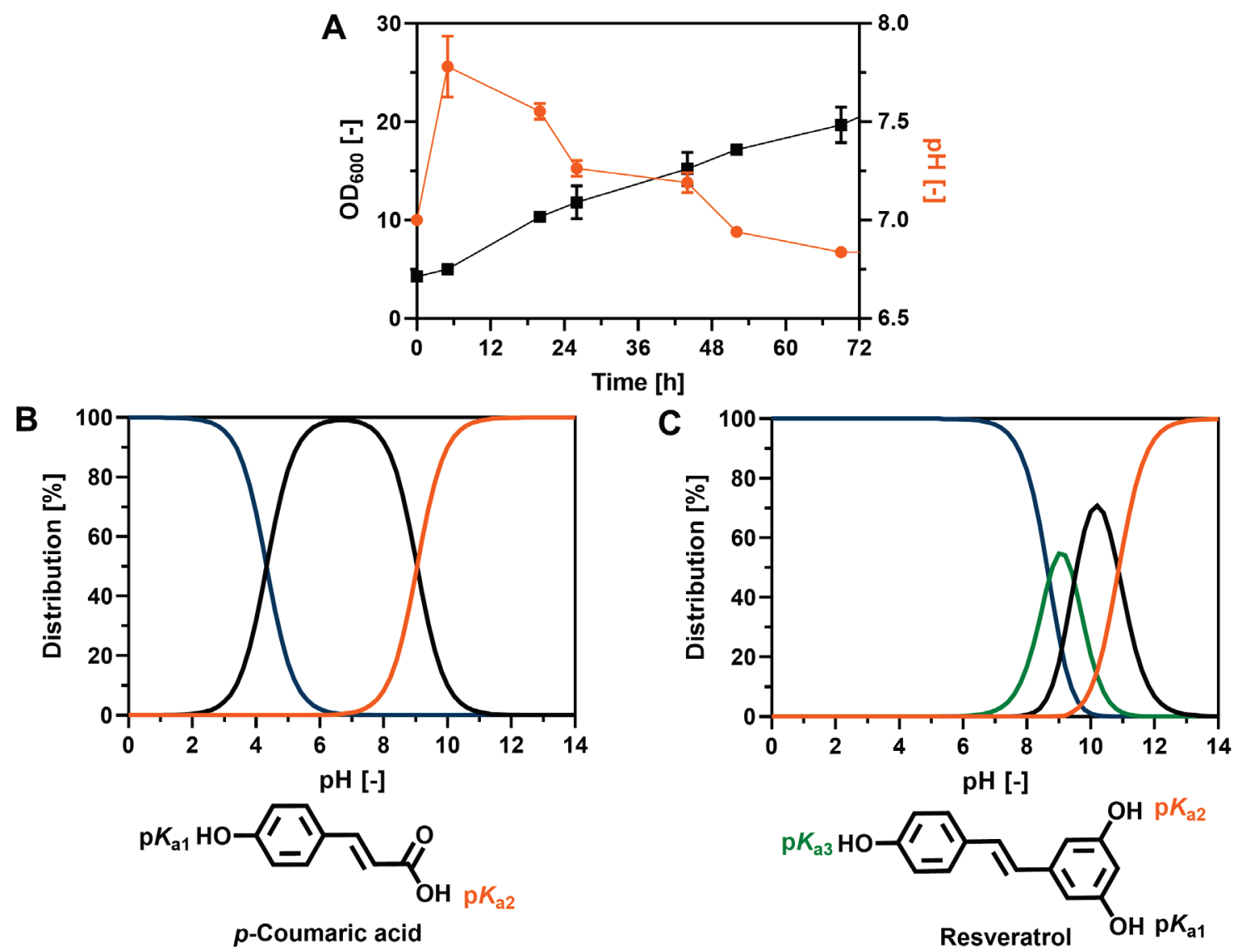

Figure S3: $\mathrm{pH}$-dependent protonation of $p$-CA and RES. A: $\mathrm{pH}$ - and $\mathrm{OD}_{600}$ profile during RES production in shake flasks using C. glutamicum-RES. Cultivation conditions: defined CGXII medium with $220 \mathrm{mM}$ D-glucose, $5 \mathrm{mM}$ $p$-CA, $1 \mathrm{mM}$ IPTG, initial $\mathrm{pH}_{0}=7$; baffled shake flask: $\mathrm{V}_{\mathrm{L}}=50 \mathrm{~mL}$ in $500 \mathrm{~mL}$ flasks, $\mathrm{T}=30^{\circ} \mathrm{C}, \mathrm{n}=120 \mathrm{rpm}$, shaking diameter $=50 \mathrm{~mm}$. B: Percentage distribution of fully protonated (blue), single protonated (black) and unprotonated (orange) $p$-CA depending on the $\mathrm{pH}$ are given. C: Percentage distribution of fully protonated (blue), double protonated (green), single protonated (black) and unprotonated (orange) RES depending on the $\mathrm{pH}$ are given. Data represent average values and standard deviation of three independent replicates. 


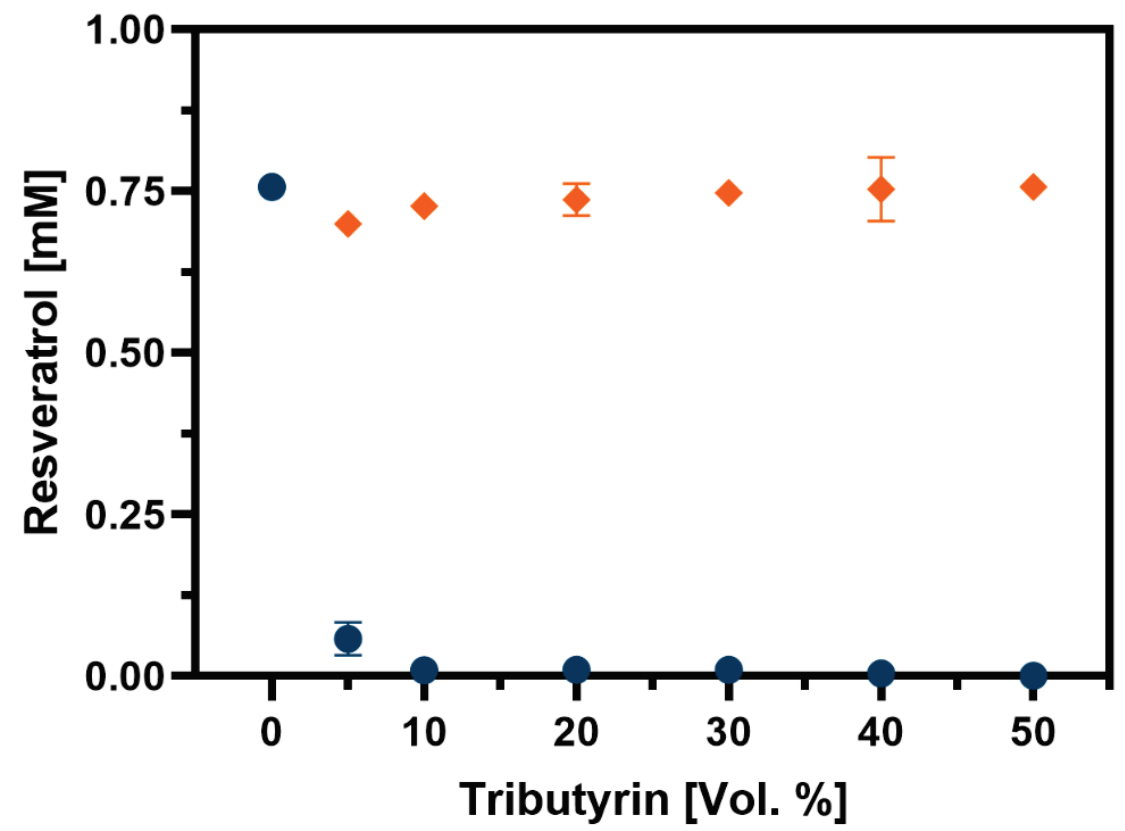

Figure S4: Investigation of tributyrin ratios for sufficient resveratrol extraction from aqueous phase (blue) into organic solvent phase (orange) at $\mathrm{pH} 7$.

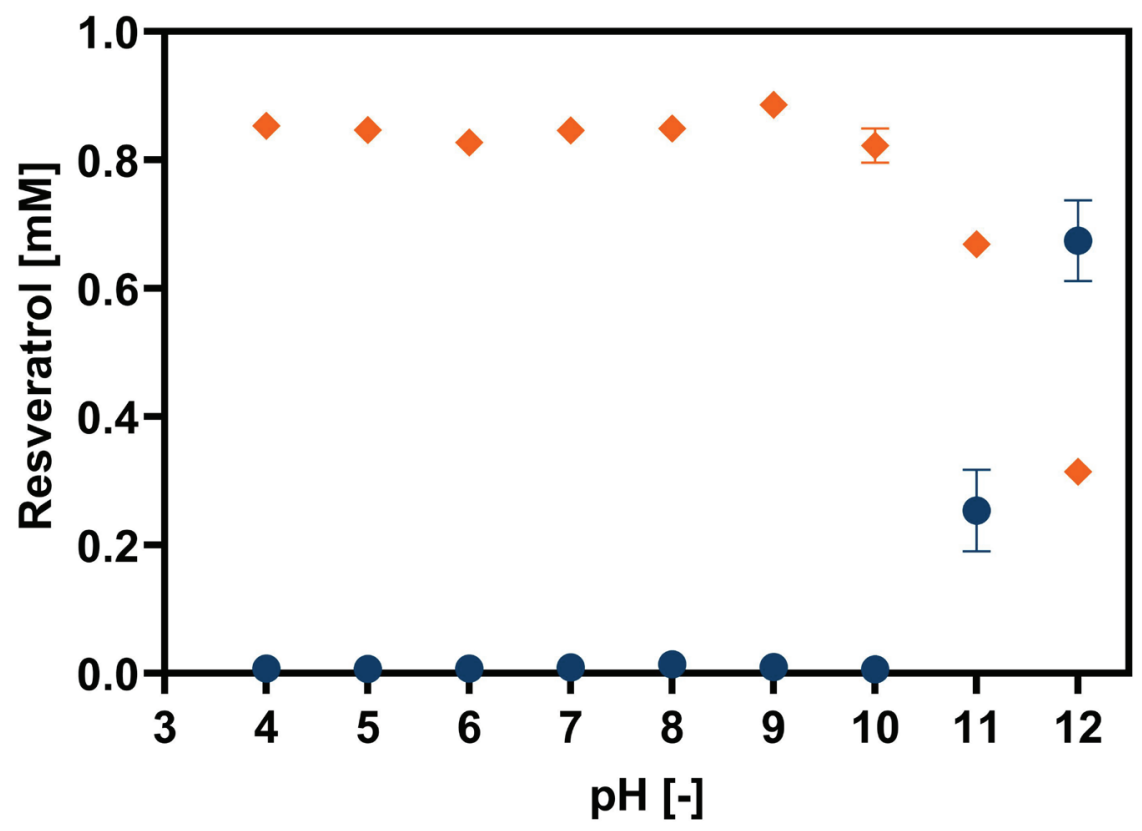

Figure S5: Back-extraction of resveratrol from TB phase (orange) into the aqueous phase (blue) at different $\mathrm{pH}$ values. Data represent average values and standard deviation of three independent experiments. 
Table S1. Organic solvents tested for the development of an in situ liquid-liquid extractive cultivation process for C. glutamicum-RES. Data sources: PubChem (National Center for Biotechnology Information, NCBI, Bethesda, MD, USA) and ChemSpider (Royal Society of Chemistry, London, UK).

\begin{tabular}{lcccc}
\hline \multicolumn{1}{c}{ Organic solvent } & $\log (\boldsymbol{P})$ & $\begin{array}{c}\text { Density } \\
{\left[\mathrm{g} \mathrm{cm}^{3-1}\right]}\end{array}$ & $\begin{array}{c}\text { Water solubility } \\
{\left[\mathrm{g} \mathrm{L}^{-1}\right]}\end{array}$ & $\begin{array}{c}\text { Boiling point } \\
{\left[{ }^{\circ} \mathrm{C}\right]}\end{array}$ \\
\hline 1-Hexanol & 2.03 & 0.85 & $5.9 \cdot 10^{-1}$ & 314.8 \\
1-Octanol & 3.0 & 0.83 & $5.4 \cdot 10^{-1}$ & 195 \\
1-Decanol & 4.57 & 0.83 & $3.7 \cdot 10^{-2}$ & 229 \\
Tributyrin & 2.54 & 1.032 & $\mathrm{n} / \mathrm{A}$ & 307 \\
Methyl decanoate & 4.41 & 0.87 & $4.4 \cdot 10^{-3}$ & 224 \\
Ethyl decanoate & 4.6 & 0.86 & $6.8 \cdot 10^{-3}$ & 136 \\
Ethyl benzene & 4.79 & 0.87 & $1.6 \cdot 10^{-2}$ & 139 \\
Xylene & 3.14 & 0.87 & $2.4 \cdot 10^{-1}$ & 174 \\
Decane & 5.01 & 0.7 & $5.2 \cdot 10^{-5}$ & 216 \\
Dodecane & 6.1 & 0.75 & $3.7 \cdot 10^{-6}$ & \\
\hline
\end{tabular}


Table S2. Performance parameters of batch and pulse fed-batch bioreactor cultivations. Data represent average values and standard deviation from two independent experiments.

\begin{tabular}{|c|c|c|c|c|}
\hline \multirow[b]{2}{*}{ Organic solvent } & \multicolumn{2}{|c|}{ Batch } & \multicolumn{2}{|c|}{ Pulse Fed-Batch } \\
\hline & Single-phase & Two-phase & Single-phase & Two-phase \\
\hline$\mu_{\max }\left[\mathrm{h}^{-1}\right]$ & $0.13 \pm 0.01$ & $0.15 \pm 0.01$ & $0.13 \pm 0.01$ & $0.16 \pm 0.01$ \\
\hline RES titer AQ phase [mM] & $0.3 \pm 0.01$ & $0.9 \pm 0.01$ & $\begin{array}{l}0.3 \pm 0.02 \\
0.92 \pm 0.02^{\mathrm{a}}\end{array}$ & $\begin{array}{l}0.1 \pm 0.01 \\
0.2 \pm 0.05^{\mathrm{a}}\end{array}$ \\
\hline RES titer OR phase $[\mathrm{mM}]$ & - & $1.2 \pm 0.01$ & $\begin{array}{l}- \\
-\end{array}$ & $\begin{array}{l}4.1 \pm 0.4 \\
7.5 \pm 0.4^{\mathrm{a}}\end{array}$ \\
\hline RES production $\left[\mathrm{mM} \mathrm{h}^{-1}\right]$ & $0.02 \pm 0.001$ & $0.06 \pm 0.001$ & $\begin{array}{l}0.01 \pm 0.01 \\
0.15 \pm 0.01^{\mathrm{a}}\end{array}$ & $\begin{array}{l}0.15 \pm 0.01 \\
0.26 \pm 0.03^{\mathrm{a}}\end{array}$ \\
\hline$p$-CA uptake $\left[\mathrm{mM} \mathrm{h}^{-1}\right]$ & $0.02 \pm 0.001$ & $0.04 \pm 0.001$ & $\begin{array}{l}0.1 \pm 0.01 \\
0.2 \pm 0.01^{\mathrm{a}}\end{array}$ & $\begin{array}{l}0.13 \pm 0.03 \\
0.26 \pm 0.02^{\mathrm{a}}\end{array}$ \\
\hline D-Glc uptake $\left[\mathrm{mM} \mathrm{h}^{-1}\right]$ & $4.6 \pm 0.2$ & $5.5 \pm 0.4$ & $4.3 \pm 0.8$ & $5.6 \pm 0.9$ \\
\hline Yield $\left(\mathrm{mmol}_{\mathrm{RES}} \mathrm{mmol}_{p-\mathrm{CA}^{-1}}\right)$ & $0.11 \pm 0.01$ & $0.36 \pm 0.09$ & $\begin{array}{l}0.16 \pm 0.01 \\
0.0 \pm 0.0^{\mathrm{a}}\end{array}$ & $\begin{array}{l}0.90 \pm 0.02 \\
0.92 \pm 0.01^{\mathrm{a}}\end{array}$ \\
\hline
\end{tabular}

a after manual $p$-CA feed 